\title{
Cooperación académica: Universidad y sector productivo regional
}

\author{
Academic cooperation: \\ University and regional productive sector \\ Cooperação acadêmica: \\ Setor produtivo universitário e regional \\ Rolando Eslava-Zapata**, Genny Chacón-Lobo ${ }^{\mathrm{b}}$, Édixon Chacón-Guerreroc ${ }^{\mathrm{c}}$, Hilário Alonso \\ Gonzalez-Júniord ${ }^{\mathrm{d}}$. \\ aDoctor en Administración de Organizaciones, Universidad de los Andes ,Mérida- Venezuela; (ID \\ ${ }^{b}$ Magister en Administración Universidad de los Andes, Mérida- Venezuela (D); ${ }^{\text {DD }}$ octor en \\ Psicología Universidad de los Andes, Venezuela (ID; d Asistente de profesor Universidad de los \\ Andes, Mérida- Venezuela. (DD \\ Forma de citar: Eslava, R., Chacón, G., Chacón, E. \& Gonzales, H. (2018). Cooperación académica: \\ Universidad y sector productivo regional. Perspectivas, 3(1). 102-114
}

Recibido: julio 08 de 2017

Aceptado: octubre 20 de 2017

\section{Palabras clave \\ Cooperación, universidad, pasantías, sector productivo regional.}

Resumen: El presente artículo tiene como propósito plantear los factores que influyen en el fortalecimiento de la cooperación académica entre la Universidad y el sector productivo regional durante el proceso de las pasantías universitarias. En tal contexto, este documento se define como un estudio de tipo descriptivo documental que procura el aporte de elementos de reflexión con respecto a la estructura económica actual y la necesaria transferencia de conocimientos por parte de los egresados universitarios a la sociedad. En este sentido, se pudo concluir que la relación sector productivo-Universidad se hace hoy prioritaria, pues de allí depende el desarrollo de la capacidad científica y tecnológica de un país. En efecto, la renovación y actualización del recurso humano con habilidades y conocimientos científicos representa la base para intervenir en una sociedad cada día más exigente y con una economía altamente competitiva. Por tanto, el binomio academia - empresa construye el fundamento teórico práctico pertinente para sustentar la relación desde el punto de vista económico, social, político, cultural y educativo en el proceso de democratización del conocimiento científico y tecnológico.

* Autor para correspondencia

rolandoeslava@ula.ve

https://doi.org/10.22463/25909215.1427 


\section{Keywords}

Cooperation, university, internships, regional productive sector.

\section{Palavras chave}

Cooperação, universidade, estágios, setor produtivo regional.
Abstract: The purpose of this article is to outline the factors that influence the strengthening of academic cooperation between the University and the regional productive sector during the process of university internships. In this context, this document is defined as a documentary descriptive study that seeks the contribution of elements of reflection with respect to the current economic structure and the necessary transfer of knowledge by university graduates to society. In this sense, it was possible to conclude that the productive sectorUniversity relationship becomes a priority today, since the development of the scientific and technological capacity of a country depends on it. In effect, the renewal and updating of human resources with scientific skills and knowledge represents the basis for intervening in a society that is increasingly demanding and with a highly competitive economy. Therefore, the academy - company binomial constructs the pertinent theoretical and practical foundation to sustain the relationship from the economic, social, political, cultural and educational point of view in the process of democratization of scientific and technological knowledge.

Resumo: O objetivo deste artigo é descrever os fatores que influenciam o fortalecimento da cooperação acadêmica entre a Universidade e o setor produtivo regional durante o processo de estágios universitários. Neste contexto, este documento é definido como um estudo documentário descritivo que busca o contributo de elementos de reflexão em relação à estrutura econômica atual e a necessária transferência de conhecimento pelos graduados universitários para a sociedade. Nesse sentido, foi possível concluir que o setor produtivo - a relação universitária se torna uma prioridade hoje, já que o desenvolvimento da capacidade científica e tecnológica de um país depende disso. Com efeito, a renovação e atualização de recursos humanos com habilidades científicas e conhecimento representa a base para intervir em uma sociedade cada vez mais exigente e com uma economia altamente competitiva. Portanto, o binômio academia - empresa constrói os fundamentos teóricos e práticos pertinentes para sustentar a relação do ponto de vista econômico, social, político, cultural e educacional no processo de democratização do conhecimento científico e tecnológico. 


\section{Introducción}

Las universidades, sean públicas o privadas, se encuentran inmersas en un entorno cada vez más cambiante. En este escenario, la necesidad de responder a las continuas demandas de productos y servicios ha hecho que la cooperación entre los sectores educativo y productivo (o empresarial) se convierta en una herramienta imprescindible para la supervivencia de cualquier organización. No obstante el reconocimiento de esta cooperación necesaria, la colaboración entre sector productivo y universidad no siempre ocurre de manera efectiva. De esta opinión es García (2006), que señala lo siguiente:

Empresas y universidades manejan poca información sobre diversidad de formas de relación que favorezca a cada institución [...]. Además, es poco o no muy extendido el manejo de redes comunicacionales por ambas partes; y por si fueran pocas las debilidades, son imprecisos o no advertidos los campos y núcleos de experimentación y excelencia, como la fuente de muchas competencias, por parte de las universidades. (p.169)

De lo anterior se infiere que la consideración de la relación entre la empresa y la Universidad actual debería constituir una de las tareas primordiales para las autoridades universitarias y empresariales. Esta relación, en efecto, influye en el comportamiento y las percepciones estabilizadas que filtran la realidad y condicionan los niveles de participación, para lo cual tendría que mejorar la vinculación de los actores involucrados. Asimismo, una relación estrecha entre empresa y universidad influye sobre la productividad, revaloriza el conocimiento como esencia y herramienta fundamental y orienta de manera más eficaz la formación integral de los estudiantes universitarios en función de las necesidades y expectativas de la organización o empresa que lo contratará. En periodo especialmente útil para evaluar esta relación es el que corresponde a las pasantías profesionales de los estudiantes universitarios durante su etapa final de carrera.

Para que exista una efectiva relación de cooperación entre el sector educativo y el productivo es necesaria la coexistencia de varios factores que, a decir de Ríos y Perozo (2007), se categorizan de la manera siguiente:

Factores estructurales, que se corresponden con condiciones y procesos del contexto nacional, regional y local del ámbito de las actividades económicas, políticas, culturales, sociales, científicas, tecnológicas. Factores institucionales, que aluden a las características, condiciones y elementos particulares e intrínsecos a la organización académica y empresarial. Factores individuales: que se localizan a nivel de las actitudes, capacidades y disposición de las personas integrantes de las organizaciones que participan en la relación ( $p$. 123).

Cada uno de estos grupos de factores se ve afectado por circunstancias potenciadoras, dinamizadoras o inhibidoras, según sea el caso, que favorecen o retardan el proceso de cooperación y en oportunidades impiden incorporar el pasante de la universidad a la empresa. Durante el periodo de pasantías, en efecto muchos estudiantes se enfrentan a dificultades de distinto grado, a pesar de que se trata de un trabajo directamente relacionado con la especialidad en estudio que tiene como finalidad proporcionar experiencia en su futuro campo laboral, en el que se aplican los conocimientos teóricos y prácticos adquiridos en la institución.

La Declaración Mundial sobre la Educación Superior de la UNESCO (1998) señala como una de las funciones de la Educación Superior la formación de profesionales altamente capacitados y responsables, para hacer frente a la totalidad de las circunstancias que caracterizan la vida humana, respondiendo con acciones calificadas, producto la combinación de conocimientos teóricos y prácticos de alto nivel, adquiridos en los cursos de estudios 
superiores y adaptados a las necesidades presentes y futuras de la sociedad. En este sentido, según la opinión de Sánchez (2005),

Los nuevos escenarios que se plantean en la sociedad del conocimiento han promovido la revisión de la pertinencia social de las universidades y la búsqueda de estrategias que permitan enfrentar la incertidumbre que los caracteriza. Una de ellas ha sido la de estrechar relaciones con el sector externo, público y privado, para la formación profesional universitaria mediante distintos tipos de actividades entre las cuales las pasantías se perfilan como las actividades más sistemáticas, pertinentes y de mayor duración que permiten a los estudiantes, en su incursión a los espacios laborales reales, contrastar la teoría y la práctica, lo académico y lo laboral y, de esta manera, desarrollar las competencias para la empleabilidad. (p. 345-357)

De este modo, las pasantías poseen un valor relevante por cuanto permiten que los estudiantes apliquen sus conocimientos teóricos y prácticos, desarrollen habilidades y destrezas y adquieran nuevos conocimientos que contribuyan con su adiestramiento. Por ello se hace necesario que el sector productivo y la universidad, de manera conjunta, atiendan la formación académica y el desarrollo humano a través de la mutua cooperación. Una oportunidad excelente para esta simbiosis está representada por las pasantías universitarias.

En relación con los planteamientos precedentes, el aporte de este artículo consiste en exponer desde una perspectiva teórica la necesidad de que la universidad se mantenga en permanente esfuerzo por resultar competitiva, por generar acciones en la organización, tales como las pasantías, que permitan incrementar la eficacia de esta etapa formativa en función de las necesidades de las empresas para las que forma a sus egresados. En este sentido, las pasantías deben concebirse como un proceso durante el cual se inicia al estudiante universitario tanto en el ejercicio profesional como en la proyección de los valores y las capacidades en la que los ha formado la Universidad en función de las expectativas de los sectores público y privado de la región.

Las circunstancias en las cuales un estudiante universitario emprende la etapa de sus pasantías profesionales divergen mucho según el lugar y el momento. En el escenario universitario venezolano actual existen dificultades añadidas, pues tal y como señala Faría (2005), las distintas perturbaciones que se dan debido a factores múltiples tales como el incremento de la inflación, la carencia de ciertos recursos formativos y el déficit presupuestario asociado a la productividad y la competitividad auténtica en relación con los avances de la ciencia y la tecnología, dificultan tal reto.

En este contexto de ideas, el propósito de este artículo consiste en plantear de manera teórica los factores que influyen en el fortalecimiento de la cooperación académica entre la universidad y el sector productivo regional durante el desarrollo de las pasantías universitarias.

En consecuencia, el alcance del presente trabajo es eminentemente teórico, puesto que se basa enun arqueo de información documental sobre los factores que intervienen en elproceso que se cumple durante el periodo de pasantías profesionales: adjudicación de pasantías, grado de cooperación académica entre el sector productivo regional y la universidad e índice de cupos en organizaciones que brindan la posibilidad de una experiencia laboral de calidad para los futuros egresados.

\section{La universidad venezolana y su relación con el sector productivo}

\subsection{Cooperación sector productivo - universidad}

La cooperación como concepto descansa, según Espinoza (1999), en la acertada concepción del "fortalecimiento y crecimiento de las organizaciones que de acuerdo a su naturaleza logren acuerdos de cooperación" (p. 176). En otras 
palabras, la cooperación está referida al enfoque desde el cual dos o más organizaciones sociales logran acuerdos, formales o informales, bajo un clima de confianza mutua con la finalidad de compartir la gestión y atendiendo a los riesgos que puedan surgir.

En este contexto, el tema de la cooperación sector productivo- universidad ha tomado particular relevancia y se ha convertido en una práctica necesaria para el progreso de las naciones a través del desarrollo del conocimiento, la capacidad científica y la tecnología (Duarte Montenegro, 2014). Esta relación trasciende el ámbito de los dos sectores, dado que alcanza dimensiones teóricas y aplicativas de carácter social, económico y educacional.

Por eso las Naciones Unidas plantean que las universidades están claramente infrautilizadas en lo que concierne a la ciencia y la tecnología. Si se fomenta la cooperación entre las universidades y la industria, las universidades serán capaces de potenciar el desarrollo regional y nacional (Guillén Mondragón, 2012).

De allí la importancia de que las universidades venezolanas se conviertan en núcleos de investigación científica y tecnológica, en estrecha relación con los sectores productivos (Montoya Ríos y Aguilar Zambrano, 2013), con la finalidad de fortalecer su capacidad para cumplir con los más altos estándares de calidad educativa, acorde con su misión como formadoras de recursos humanos.

La cooperación también puede definirse como un proceso en el que las organizaciones logran acuerdos, persiguen sus propias metas y orientan sus acciones hacia resultados comunes (Paz, 2011). Así, la Corporación para el Desarrollo (2001), citada por Mujica (2001), señala que la cooperación sector productivo - universidad:

Es el conjunto de actividades específicamente orientadas a perfeccionar los recursos humanos locales y la capacidad de las instituciones vinculadas al desarrollo, por medio de la transferencia de conocimientos, experiencias, aptitudes, habilidades productivas, gerenciales y la capacidad de dominio técnico y tecnológico. (p. 99)

De esta manera, la cooperación se consolida como una vía para lograr que ambos sectores, empresarial y educativo, alcancen el éxito a través de la combinación y explotación conjunta de sus capacidades y recursos, lo cual no solo favorece el mejoramiento de las empresas y las universidades, sino de la economía regional, nacional y de la sociedad en general. El cuadro 1 resume los efectos de la cooperación sector productivo-universidad.

Cuadro 1

Efectos de la cooperación sector productivo- universidad.

\begin{tabular}{ll}
\hline \multicolumn{1}{c}{ Para la empresa } & \multicolumn{1}{c}{ Para la universidad } \\
\hline $\begin{array}{l}\text { Genera un flujo de personal desde la empresa a la universidad } \\
\text { (profesionales) que transmitirán sus conocimientos prácticos y } \\
\text { renovarán sus conocimientos teóricos. }\end{array}$ & $\begin{array}{l}\text { Facilita la creación de equipos de investigación mixtos que } \\
\text { avanzan conjuntamente de una forma más eficaz y directa que } \\
\text { por separado. }\end{array}$ \\
$\begin{array}{l}\text { Crea un soporte técnico e investigador que permite mantener el } \\
\text { contacto con el mundo científico y de la tecnología. }\end{array}$ & $\begin{array}{l}\text { Da lugar a un flujo de personal desde la universidad a la } \\
\text { empresa (profesores e investigadores) derivando en } \\
\text { conocimientos que contribuyen al mejoramiento de la práctica } \\
\text { profesional en el ámbito universitario. }\end{array}$ \\
& $\begin{array}{l}\text { Genera ingresos adicionales que suelen beneficiar a la } \\
\text { comunidad universitaria. }\end{array}$ \\
& $\begin{array}{l}\text { Financia la compra de materiales y equipos necesarios para el } \\
\text { mejoramiento de la labor académica. }\end{array}$ \\
\hline $\begin{array}{l}\text { Integra a las universidades en el entorno económico y social en } \\
\text { el que desenvuelven su labor. }\end{array}$ \\
\hline
\end{tabular}


Dados que estos efectos pueden calificarse como positivos, el sector productivo y la Universidad están asumiendo la responsabilidad de formalizar sus relaciones por medio de alianzas y acuerdos para desarrollarse y mantenerse coherentes y pertinentes con el entorno en el cual se desenvuelven.

Washmuth (2009) ordena las formas de cooperación entre el sector productivo y la Universidad en cuatro áreas típicas: (a) fomento a la infraestructura científica, (b) cooperación y financiamiento para la investigación, (c) intervención en el estudio y perfeccionamiento, $\mathrm{y}$ (d) apoyo a estudiantes de pregrado y postgrado.

El fomento a la infraestructura científica comprende todas las actividades que favorecen la enseñanza académica y a la investigación. Entre ellas se encuentran las donaciones a las universidades, patrocinio de puestos para profesores, cofinanciación de estructura física, de equipamiento, de asociaciones de egresados y de diversos eventos. El área de cooperación y financiamiento para la investigación involucra los proyectos conjuntos de investigación, la fundación de institutos, donaciones para jornadas científicas y aportes a premios científicos. Por su parte, la intervención en el estudio y perfeccionamiento se relaciona con la sociedad para el establecimiento de programas de estudio duales y programas de componentes prácticos, la contribución en el diseño de planes de estudio y el fomento a la oportunidad de empleo para los graduados a través de la creación conjunta de carreras. Finalmente, el apoyo a estudiantes de pregrado y postgrado puede darse en forma de becas o préstamos para el estudio. También pertenecen a esta área las prácticas de los estudiantes en empresas y otras organizaciones (pasantías industriales), los programas de integración de practicantes, los programas de aprendizaje en acompañamiento al estudio, la elaboración de trabajos dentro de las empresas y el otorgamiento de premios a los mejores trabajos de grado.

Como se observa, existe una amplia variedad de formas de cooperación con el sector productivo y/o empresarial, en cada una de las cuales cada sector aporta algo y espera ciertos resultados a cambio. La más tradicional de las cuatro, y que no supone costo directo del entorno socio-económico, es la preparación del capital humano en diversas disciplinas que, al incorporarse al mercado de trabajo, solventa problemas empresariales relacionados con la manufacturera, la actividad social, cultural, habitacional, salud, agrícola, gerencial y muchas otras disciplinas que las universidades se imparten. En efecto, la mayor parte de estos rubros está relacionada con las actividades académicas, de consultoría y de apoyo a la industria que ofrecen las universidades a la empresa a través de pasantías estudiantiles y de personal en año sabático.

\subsection{Impacto de la cooperación sector productivo - universidad en las pasantías universitarias}

Con respecto a la naturaleza de la relación sector productivo-universidad habrá quien suponga que la universidad procura fundamentalmente financiamiento para sus investigaciones; la realidad, sin embargo, es distinta y está superando estas expectativas primarias de las empresas. Entre los factores que ofrecen las instituciones académicas al sector productivo se encuentran el suministro de personal capacitado, la concreción de conocimientos científicos exitosos y la obtención e intercambio de recursos para satisfacer las demandas del medio ambiente, asegurando la permanencia de las empresas en el tiempo, visualizando la relación como un negocio. Las universidades buscan, además, poder integrar la investigación académica a la empresa, con la finalidad de ampliar sus oportunidades y fortalecerse como organización (Andreozzi, 2011).

La acelerada obsolescencia del conocimiento hace necesario el desarrollo de nuevas habilidades y destrezas intelectuales; es allí donde la relación sector productivo-universidad emerge como respuesta a las demandas del sector industrial. En efecto, es de las universidades de donde surgen fundamentalmente los aprendizaje, el entrenamiento, la formación y capacitación del recurso humano necesarios para la 
producción eficaz de bienes y servicios, así como al establecimiento de estrategias necesarias para desarrollar ambas organizaciones en el entorno donde se desenvuelven para la generación de riqueza (De La Vega y Arakaki, 2011).

En relación con lo anterior, la puesta en marcha de programas responsables de pasantías y de prácticas profesionales supervisadas constituyen mecanismos viables de fomento, mantenimiento $\mathrm{y}$ fortalecimiento de interacciones estables y mutuamente benéficas (Macías Mozqueda, 2011). En cuanto a la necesidad de formar profesionales cónsonos con los adelantos tecnológicos y científicos actuales, las universidades deben asegurar que el sistema de educación y capacitación forme a personas que puedan adaptarse a las circunstancias cambiantes del mercado laboral, y desarrollen aptitudes y competencias para desempeñarse en la industria (Becerra Bizarrón, Zúñiga Bobadilla, Vargas Aceves, Alegría, y Malacara Castillo, 2014).

Por esta razón, es indudable la importancia que tiene para el país el desarrollo de una educación con un nivel de calidad, apoyada en los beneficios que aporta la cooperación con el sector productivo (Rodríguez Quezada, Cisterna Cabrera, y Gallegos Muñoz, 2011), que sirva de apoyo a los nuevos retos que impone el futuro, pues si se quiere llevar el país al desarrollo económico y social, es necesaria la formación de un egresado cuya mano de obra calificada, propicie su participación activa, en el éxito de la economía nacional (Jiménez Jiménez, Fandiño Cuca, Urrego Montañézy Morales Rubiano, 2012 ), para que a través de sus conocimientos, adiestramiento y habilidades enfrente el proceso de crecimiento económico y social del país.

\subsection{Factores que influyen en la cooperación sector productivo- universidad}

Como toda relación, la cooperación entre el sector productivo y la universidad, está influenciada por un conjunto de factores internos y externos que intervienen favoreciendo o dificultando su normal desenvolvimiento $\mathrm{y}$, consecuentemente, afecta el logro de los objetivos previstos.

Los factores externos están relacionados con las situaciones de orden político y económico de una sociedad. Para Ríos y Perozo (2007: 123-148), estos factores son:

a) Una asignación presupuestaria suficiente para el buen funcionamiento de la institución universitaria que le permita a esta no descuidar ninguna de sus funciones en docencia, extensión e investigación.

b) La existencia de una legislación cónsona con el escenario mundial que estimule y/o fomente dicha relación para que ambos actores puedan desempeñarse libremente y ajustados a derecho, sus roles, y a su vez, puedan obtener los beneficios que cada cual persigue (Di Meglio, y Harispe, 2015). Entre estas leyes estarían las que se relacionan con el trabajo, el régimen fiscal, las inversiones y la educación superior.

c) Un sector empresarial dispuesto al aprendizaje organizacional e interesado en mejorar su nivel competitivo y su productividad, así como en los resultados a mediano y largo plazo, en producir bienes y servicios orientados a brindar bienestar social, en primer lugar, y no sólo cuantiosas ganancias para el empresario.

Los factores externos generalmente no son controlables por el sector universitario; en el mejor de los casos, la universidad, habrá de diseñar mecanismos con la finalidad de ajustarse a los efectos que estos factores puedan producir en cualquier convenio de cooperación.

Por otra parte, se encuentran los factores internos atribuibles a la universidad, de los cuales depende la concreción y el funcionamiento de los convenios de cooperación. Entre ellos se encuentran:

a) Una Universidad preocupada por formar el profesional idóneo que requiere el país para su desarrollo económico.

b) Capacidad para brindar soluciones propias a 
los problemas que afectan a la sociedad.

c) Posibilidad de mantener un personal adecuado, en cuanto a número y capacitación, para gestionar en esa relación de cooperación.

d) Mecanismos de control que permitan la realización coordinada de las actividades entre las partes.

e) Disposición de los recursos financieros para solventar las actividades que han de desempeñarse conjuntamente.

Así pues, antes de poner en marcha un convenio de cooperación entre el sector productivo y la universidad, es necesario que ambas instituciones realicen un estudio previo de sus capacidades, teniendo en cuenta las condiciones internas y externas que intervienen en el proceso, a los fines de generar conocimiento y beneficios tanto para ambas partes como para la sociedad (Bautista, 2014).

Espinoza (1999), citado en Ríos y Perozo (2007) propone la identificación y organización de estos factores tomando en cuenta una doble condición: La primera es su definición por el contexto y/o nivel de pertenencia, que corresponde a tres categorías: a) factores estructurales, b) factores institucionales y c) factores individuales. La segunda condición es su definición por el alcance del efecto: a) factores potenciadores, b) factores dinamizadores y c) factores inhibidores. La relación de estos factores se presenta en el cuadro 2.

Cuadro 2

Factores que inciden en la cooperación sector productivo-universidad.

Factores potenciadores

*Avance del conocimiento

*Turbulencia económica

Factores Estructurales
*Expectativas y presión social hacia la universidad.

Factores dinamizadores

* Políticas gubernamentales para elevar la capacidad nacional de exportación de avances científicos y tecnológicos.

* Políticas gubernamentales para subsidiar programas de vinculación.

*Políticas comerciales que favorezcan la instalación de industrias y empresas cercanas al área universitaria.

\section{*Imperativos económicos}

Factores Institucionales
*Necesidad de diversificar fuentes para financiar la *Infraestructura para la actividad de investigación $y$ transferencia de ciencia $y$ desarrollo de la empresa.

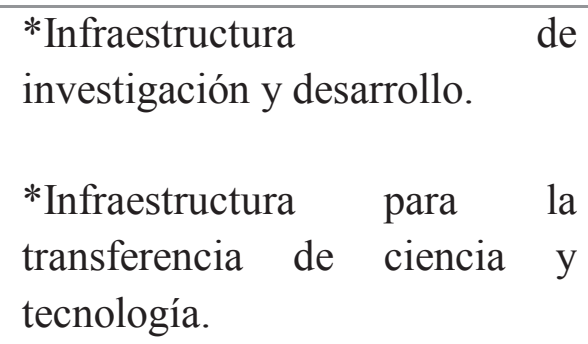




\begin{tabular}{|c|c|c|}
\hline & $\begin{array}{l}\text { *Detección de nichos en el } \\
\text { mercado para colocar bienes y } \\
\text { servicios universitarios. }\end{array}$ & $\begin{array}{l}\text { *Sistema de incentivos } \\
\text { académicos y económicos para } \\
\text { los investigadores. }\end{array}$ \\
\hline \multirow{3}{*}{ Factores Individuales } & $\begin{array}{l}\text { Motivaciones emprendedoras de } \\
\text { los académicos. }\end{array}$ & $\begin{array}{l}\text { *Acceso a financiamiento de } \\
\text { proyectos de investigación. }\end{array}$ \\
\hline & $\begin{array}{l}\text { *Actitud innovadora de los } \\
\text { ejecutivos empresariales. }\end{array}$ & $\begin{array}{l}\text { *Obtención de } \\
\text { adicionales. }\end{array}$ \\
\hline & & $\begin{array}{l}\text { *Participación } \\
\text { industrialización } \\
\text { tecnologías. }\end{array}$ \\
\hline
\end{tabular}

Fuente: Elaboración propia (2017)

Se trata entonces, de orientar las acciones de manera que el binomio sector productivouniversidad pueda consolidar las organizaciones con capital intelectual que se convierta en mediador de la relación, favoreciendo dicha interacción. Esto es fundamental ya que una universidad y una empresa almacenan conocimiento de muchos niveles, conocimiento que se debe compartir por intereses comunes y de la sociedad en la cual se desenvuelven.

\subsection{Relación universidad-sector productivo}

En el mundo de la globalización, donde el libre comercio constituye la punta de lanza de la comercialización entre países, la relación entre la universidad y el sector productivo, desencadena el surgimiento de una serie de consideraciones en cuanto a los valores, fines y medios que se articulan para la materialización de tal hecho. Al respecto Tasker y Packhman(1993), señalan que comúnmente se asume que los valores predominantes del sector productivo están determinados por motivos de utilidad y beneficios; mientras que los valores que están ampliamente sustentados en las universidades están definidos por los principios de amplia indagación y libertad intelectual.

En concordancia con lo anterior, Schavino de Viloria (1999) afirma que axiológicamente las universidades se orientan hacia la formación de talento humano, la gestión del saber, la intelectualidad en contextos académicos y el crecimiento científico, mientras que las organizaciones del sector productivo apuntan hacia la producción de bienes y servicios, la obtención de beneficios económicos, la comercialización y la rentabilidad.

Por estas razones, para alcanzar una adecuada vinculación universidad - sector productivo, debe establecerse un puente axiológico, orientado hacia el beneficio mutuo, el mejoramiento continuo, la excelencia, la competitividad, la productividad, la solución de problemas y la satisfacción de necesidades (Di Meglio, 2017).

En este orden de ideas, la forma de direccionar la relación entre la universidad y el sector productivo trasciende los intereses particulares de cada uno. El escenario que se tiene de una sociedad y de una nueva economía global, donde los cambios constantes son esenciales para poder competir exitosamente, colocan a la Universidad y al sector productivo en 
un contexto donde las alianzas estratégicas son de fundamental conveniencia para enfrentar las fuerzas que regulan y modelan tanto la actividad económica como las de naturaleza social, política, científica $\mathrm{y}$ educacional en los espacios internacionales, nacionales y regionales.

Ahora bien, debido a la dinámica de los mercados y la producción, es inevitable y necesario que los países tengan que hacer un uso más racional y eficiente del capital intelectual de las universidades (Wasmuth, 2009) como parte de su esfuerzo para competir efectivamente en el mercado mundial y generar un crecimiento en la conciencia pública de que la revitalización de la economía puede ser solamente lograda si todos los sectores de la sociedad actúan conjuntamente. El desarrollo económico, en efecto, es un proceso de innovación que incrementa la capacidad de los individuos y las organizaciones para producir bienes y servicios y, en consecuencia, crear riqueza. La participación ideal de la Universidad en el desarrollo económico se expresa en el uso estratégico de los recursos basados en el conocimiento para sustentar el desarrollo de la economía local, estadal y regional.

Considerando en este entorno de la economía, se señalan algunas funciones de la universidad en el sector productivo que tocan su relación con el sector productivo para adquirir capital y beneficios económicos. Espinoza (1999) destaca las siguientes:

a) La primera función tiene que ver con los tradicionales objetivos de calificar y desarrollar recursos humanos (Rodríguez Pérez, Acuña Martínez, Rojas Vera y Lobato, 2015). El papel que debe cumplir la educación en el proyecto de desarrollo de la sociedad parte de la asunción de que el conocimiento es actualmente el verdadero capital y el principal recurso para la producción de riqueza de los países. Por tanto, contra los criterios manejados hasta el momento, no son los recursos naturales y los capitales monetarios los que determinarán el nivel de desarrollo posible, sino el conocimiento y la capacidad de obtener y manejar información. b) Para alcanzar posiciones ventajosas en un contexto de globalización y de transformaciones en los ámbitos técnico, económico y sociocultural, la educación debe estar dirigida a formar un capital humano altamente calificado. Es importante precisar que los cambios que se están dando en el ámbito técnico-económico, implican una transformación profunda en las formas y dinámicas de producción, que requieren de organizaciones que se adapten permanentemente a los cambios, que estén constituidas por trabajadores creativos, capaces de innovar, de incorporar aprendizajes a sus labores cotidianas, de tomar decisiones y de obtener y manejar información (Ruiz y Alba, 2013).

c) La productividad de una empresa y la capacidad competitiva de un país están asociadas con la calificación y formación de sus trabajadores, vinculado entonces al perfil laboral de los egresados de las instituciones educativas (Vega Jurado, Manjarrés Henríquez, Castro Martínez y Fernández de Lucio, 2011), en este caso específico, de las universidades.

\subsection{El papel de la educación en el sector productivo}

Un elemento de vital importancia para sustentar la conveniencia de la relación entre la universidad y el sector productivo tiene que ver con el proceso de aprendizaje para la formación y desarrollo de los nuevos profesionales. En este sentido, Coldstream (1994) afirma que

El desarrollo de la noción de asociación entre la industria y la educación presenta muchas cuestiones en relación a la naturaleza de la enseñanza de pregrado. Esa asociación no es tan simple como una entre clientes y suplidores, y no se trata tampoco de producir graduados respecto a un estándar de especificación conocido. Más que eso, se trata de que ellos desarrollen las habilidades en la Educación Superior inicial para continuar aprendiendo a través de sus crecientes vidas de trabajo complejas (p. 44).

De acuerdo con el autor, las organizaciones y la tecnología cambian tan rápidamente que incluso se 
ha establecido que el conocimiento técnico tiene un promedio de vida de siete años. Ante estos cambios frecuentes, las empresas necesitan personas que tengan la capacidad para aprender y desarrollar, para adaptarse a las necesidades de la organización. En cada uno de los contextos de formación de profesionales en educación superior, una constante es la demanda de habilidades transferibles (RicKap, 2012). Las más valoradas en la actividad industrial, comercial y en la actividad profesional son las habilidades intelectuales y sociales transferibles. Tanto para las instituciones educacionales como para los empleadores, las demandas proliferan en cuanto a la preparación de los estudiantes con las características adecuadas a los nuevos lugares de trabajo (Espinoza, González, y Loyola, 2011).

Al respecto, según lo señala Filmus (1996), la elevación del nivel de complejidad de las actividades genera la creación de una mayor cantidad de puestos de trabajo que requieren más capacitación para realizar operaciones con nuevas tecnologías. Para satisfacer esta necesidad, es imprescindible educar con base a una sólida formación integral (Palmar, 2013) a fin de forjar la capacidad de pensamiento teórico y abstracto y a una comprensión global del proceso tecnológico, fortalecida por una sólida formación lógico-matemática, estadística e informática.

\section{Consideraciones finales}

Tomando en cuenta el marco teórico anterior, se estima considerar la participación de representantes del sector productivo en el que debe aparecer como abanderado el sector industrial en el espacio universitario, para la identificación de las necesidades que deben satisfacer los diseños curriculares en las universidades venezolanas, en atención no solo a los conocimientos técnicos, sino también a las habilidades intelectuales fundamentales para el funcionamiento de las organizaciones en estos nuevos escenarios.

Bajo la anterior consideración, sereiterada la afirmación de en el futuro y en cualquier lugar del mundo el bienestar de la humanidad dependerá del desarrollo de la actividad económica, con base en una alta productividad y eficiencia, soportada por una creciente innovación tecnológica.

En razón de lo anterior, se hace necesario reflexionar sobre la existencia de factores internos y externos que influyen el fortalecimiento de la cooperación académica y el sector productivo. Resulta impostergable, por tanto, la consolidación de acuerdos estratégicos entre la universidad y el sector productivo para concretar la satisfacción del reto para el cambio. En esta orientación, la realidad científica, social y económica demanda el establecimiento de vínculos estrechos entre los centros generadores de conocimientos y los sectores productivos que los transforman en tecnología.

Como elemento asociado a las ideas anteriores, la concreción del puente vinculante entre ambos sectores constituye una posibilidad viable de autogestión financiera para las universidades, en un momento histórico en el que el insumo más valioso es el conocimiento y donde el desarrollo económico se perfilará básicamente por la capacidad de innovación tecnológica de las sociedades.

\section{Referencias}

Andreozzi, M. (2011). Las prácticas profesionales de formación como experiencias de pasaje y tránsito identitario. Archivos de Ciencias de la Educación, 5(5): 99-115.

Bautista, E. G. (2014). La importancia de la vinculación universidad-empresa-gobierno en México. Revista Iberoamericana para la Investigación y el Desarrollo Educativo, 5(9): $1-22$.

Becerra Bizarrón, M. E.; Zúñiga Bobadilla, L. I.; Vargas Aceves, M. Del C.; Alegría, N. L. y Malacara Castillo, A. R. (2014). Vinculación Empresa - Universidad - Gobierno, Clave en la formación integral de los egresados de las IES Mexicanas. International Review of Business Research Papers, 10(3), 16 - 31.

Coldstream, P. (1994). Training Minds for 
Tomorrow: A. Shared Responsability. HighierEducationQuarterly, 48(3): 159-168.

Chacón, G. (2014).Estrategias para el fortalecimiento de la cooperación académica entre el sector productivo regional y laUNEFA, núcleo Táchira: proceso de las pasantías. Trajo especial de grado no publicado para obtener el título de Magister. Universidad de los Andes, Venezuela.

De La Vega, A. y Arakaki, M. (2011). Las prácticas preprofesionales en la formación en Ciencias de la Información: el caso de la Pontificia Universidad Católica del Perú (PUCP). Revista Interamericana de Bibliotecología, 34(1): 77-86.

Di Meglio, M. F. y Harispe, A. (2015). Estrategias institucionales de "vinculación universitaria" orientadas al desarrollo local. Reflexiones a partir de un estudio de caso. Interações, 16(1): 203-217.

Di Meglio, M. F. (2017). Estrategias de interacción universidad-sectores productivos:Antecedentes y situación actual en las universidades de gestión estatal argentinas (1990-2015). Propuesta Educativa, 1(47): 108-118.

Duarte Montenegro, J. E. (2014). Vinculación universidad-sector productivo en el marco de las innovaciones tecnológicas. Entretemas. Revista Venezolana de Investigación Educativa, 2(1): 15-58.

Espinoza, R. (1999). Naturaleza y alcance de la relación universidad / sector productivo. Venezuela: LUZ.

Espinoza, O.; González, L. E. y Loyola, J. (2011). Relaciones entre las universidades públicas y los gobiernos para el fortalecimiento de la gestión pública en Iberoamérica. Revista del CLAD Reforma y Democracia, 50: 5-44.

Faria, H. (2005). Para rescatar la Universidad. Caracas: Cedice Libertad.

Filmus, L. (1996). The University - Industry. Relationship in Sciencie and Technology. México: Prentice.

García, O. (2006). Competencias gerenciales en la relación sector productivo-universidad como iniciativa de integración para el desarrollo.
Revista Visión Gerencial, 5(2):166-177.

Guillén Mondragón, I. (2012). La vinculación profesional universidad - sector productivo: una responsabilidad social compartida. Ingeniería Industrial. Actualidad y Nuevas Tendencias, III(9), 7-16.

Jiménez Jiménez, D. C.; Fandiño Cuca, F. A.; Urrego Montañéz, K. G. y Morales Rubiano, M. E. (2012). La contribución de las pasantías universitarias en el desarrollo decompetencias profesionales en ciencias económicas. Revista Academia y Virtualidad, 5(1): 155-170.

Macías Mozqueda, E. Y. (2011). Significado de las prácticas profesionales. La experiencia de un grupo de alumnos de nutrición de la Universidad Guadalajara Lamar. Revista Iberoamericana de Educación, 59(3): 1-10.

Mujica, M. (2001). La cooperación, una estrategia viable para relacionar a la universidad con el sector productivo. Multiciencias, 1(2):95-106.

Montoya Ríos, F. J. y Aguilar Zambrano, J. J. (2013). la relación universidad-empresa en las prácticas empresariales: Un modelo conceptual desde las técnicas de generación de ideas. Journal of Technology Management \&Innovation, 8: 196208.

Palmar, L. (2013). Barreras de comunicación interna universitaria en el desarrollo de los procesos para la vinculación con el sector productivo. Redhecs, Revista Electrónica de Humanidades, Educación y Comunicación Social, 14(8): 6171.

Paz, M. A. (2011). La necesidad de integrar el sector educativo con el sector productivo para formar profesionales competitivos en administración de empresas. Gestión y Desarrollo, 8(2): 99-122.

RicKap, C. (2012). La vinculación de la universidad con el sector productivo. Transferencia tecnológica. Ecos de Economía, 16(34): 127149.

Ríos, M. y Perozo, M. (2007). Factores que Inciden en la creación de una unidad de gestión de capital intelectual en el Núcleo LUZ-COL. Revista Venezolana de Análisis de Coyuntura, 
13(2):123-148.

Rodríguez Pérez, M. A.; Acuña Martínez, J. A.; Rojas

Vera, L. R. y Lobato, L. A. (2015). Vinculación universidad - empresa- estado, en Nicaragua. ORBIS, Revista Científica Electrónica de Ciencias Humanas, 31(11): 15-34.

Rodríguez Quezada, E.; Cisterna Cabrera, F. y Gallegos Muñoz, C. (2011). El sistema de prácticas como elemento integrante de la formación profesional. Revista de Educación Superior, XL3(159): $67-85$.

Ruiz, S. L. y Alba, C. Y. (2013). Vinculación de la academia con el sector tecnológico. Revista Matices Tecnológicos, 5, 37-41.

Sánchez, M. (2005). El aprendizaje en contextos laborales reales: El caso de las pasantías de los estudiantes universitarios. Universidad Nacional Experimental Francisco de Miranda, Falcón. Revista Venezolana de Educación, 9(30):345357.

Schavino de Viloria, N. (1999). Investigación universitaria y sector productivo. un modelo de correspondencias. Tesis Doctoral no publicada. Caracas: Universidad Santa María.

Tasker, M., y Packham, D. (1993). Industry and Higher Education: A Question of Value. HigherEducationQuarterly, 18(2): 127-136.

UNESCO, (1998). Declaración Mundial sobre la Educación Superior en el Siglo XXI. [Documento en línea]. Disponible:http://www.unesco.org/ education/educprog/wche/declaration_spa.htm.) [Consulta: 2017, Noviembre 20].

Vega Jurado, J.; Manjarrés Henríquez, L.; Castro Martínez, E. y Fernández de Lucio, I. (2011). Las relaciones universidad-empresa: tendencias y desafíos en el marco del Espacio Iberoamericano del Conocimiento. Revista Iberoamericana de Educación, 57: 109-124.

Wasmuth, W. (2009). Estado de situación de la vinculación sector productivo-universidad en Centroamérica. Alemania: Programa Universidad-Empresa para el Desarrollo Sostenible (PUEDES). 